\title{
Case series on uterine rupture depicting the atypical presentations in the labour room
}

\author{
Srishti Aggarwal*, Monika Jindal, Santosh Minhas
}

Department of Obstetrics and Gynaecology, Maharishi Markandeshwar Medical College and Hospital, Solan, Himachal Pradesh, India

Received: 18 October 2021

Accepted: 10 November 2021

\section{*Correspondence:}

Dr. Srishti Aggarwal,

E-mail: srishti.aggarwal21314@gmail.com

Copyright: (c) the author(s), publisher and licensee Medip Academy. This is an open-access article distributed under the terms of the Creative Commons Attribution Non-Commercial License, which permits unrestricted non-commercial use, distribution, and reproduction in any medium, provided the original work is properly cited.

\begin{abstract}
Uterine rupture is the complete division of all the three layers of uterus. Most uterine ruptures occur during labor in pregnant women, most commonly seen in previously scarred myometrium. Consequences of uterine rupture depend on the time between diagnosis of uterine rupture and intervention, and can be as grave as fetal and maternal death. Vigilance and avid action by the obstetrician can lead to better outcomes. Case 1 represented a 28 -year-old moderately anemic G4P2L2A1 having previous 2 LSCS at POG 39 weeks 1 day presented in COVID emergency in active labour and was found to have a uterine scar rupture $(5 \mathrm{~cm}$ rent) extending towards bladder wall with shoulder presenting on rent. A live female baby with thick meconium staining was delivered and uterine repair along with bilateral tubectomy was performed. Case 2 represented a 21-year-old primigravida with breech presentation at a gestation of 34 weeks 6 days with preterm labour pains who had been referred to our centre. Decision for LSCS was taken and on entering the abdomen rupture uterus with an inverted T-shaped rent in the upper segment extending up to the fundus was seen. A stillborn male fetus was delivered through the rent, followed by successful uterine repair. In spite of massive blood loss, the mother had survived. Case 3 represented a 30-year-old grand multipara at a gestation of 38 weeks 3 days with ultrasound documented fetal demise with fetal hydrocephalus and holoprosencephaly with labour pains was taken up for laparotomy due to suspicion of uterine rupture based on examination findings. Intra-operatively, baby was found lying in the peritoneal cavity with an unsalvageable uterus with a rupture in lower uterine segment and left lateral wall extending upto round ligament above and cervix below. A stillborn male fetus was delivered and peripartum subtotal hysterectomy with left salpingoophorectomy and right salpingectomy was done with a good maternal outcome. The above series suggest that the signs and symptoms of uterine rupture are usually variable and nonspecific, hence posing a challenge for the diagnosis. Early diagnosis and timely intervention by the obstetrician, can help us to improve the fetal and maternal outcome drastically.
\end{abstract}

Keywords: Atypical presentation, Fetal malpresentation, Hydrocephalus, Rupture, Scarred uterus, Unscarred uterus

\section{INTRODUCTION}

Uterine rupture is the complete division of all the three layers of uterus: the endometrium, myometrium, and perimetrium. ${ }^{1}$ It is a rare obstetric complication associated with severe maternal and neonatal morbidity and mortality. Though extremely rare, rupture of the nonpregnant uterus has also been reported when the uterus is exposed to trauma, infection, or cancer. ${ }^{2}$
Most uterine ruptures occur in pregnant women. Among pregnant women, there are two groups at risk for uterine rupture: those with myometrial scar from previous surgery and those with an unscarred uterus.

In developed countries uterine ruptures are mostly secondary to prior cesarean section. Risk factors include high parity, previous cesarean delivery, previous surgical evacuation of the uterus, induction and or augmentation of 
labor, malpresentation, prolonged labor, fundal pressure used to try to accomplish vaginal delivery, type of the delivery and large birth weight. ${ }^{3}$ The incidence of uterus rupture in general (primigravida or multigravida) is significantly higher in developing countries than in developed countries because of poor antenatal and obstetric care, large number of deliveries being conducted by unskilled birth attendants, prolonged unsupervised labor, and grand multiparity due to preference for male gender and illiteracy.

While the incidence of unscarred uterine rupture is low, the rate has risen considerably to one rupture per 10,000 to 25,000 deliveries. ${ }^{1}$ It is more difficult to diagnose as the patient usually presents with atypical symptoms and suspicion is low. The rupture of an unscarred uterus is also associated with significantly more maternal and neonatal morbidity than the rupture of a scarred uterus. ${ }^{4}$

Consequences of uterine rupture depend on the time between diagnosis of uterine rupture and intervention. Fetal consequences usually observed are admission to neonatal intensive care unit, fetal hypoxia, stillbirth and intrauterine death. Maternal consequences can be haemorrhage, hypovolemic shock, bladder, bowel and ureteric injury, need for hysterectomy, and as grave as maternal death. On the other hand, morbidity and mortality following rupture of the uterus depend entirely on the level of medical care. Vigilance and avid action of the obstetrician can lead to better outcomes.

Here is a series of three cases of uterine rupture along with feto-maternal outcomes presented at Maharishi Markandeshwar Medical College and Hospital, Solan.

\section{CASE SERIES}

\section{Case 1}

\section{Uterine rupture in scarred uterus}

A 28-year-old moderately anaemic G4P2L2A1 at POG 39 weeks 1 day with previous 2 LSCS had presented to the emergency with active labour pains. On examination, she had cephalic presentation, uterine contour was maintained and patient had adequate uterine contractions with a cervical dilatation of $6 \mathrm{~cm}$. Fetal heart was present with reactive NST. Emergency caesarean under general anaesthesia was decided in view of previous two LSCS in active labour. On opening the abdomen, scar rupture $(5 \mathrm{~cm}$ rent) extending towards bladder wall with shoulder presenting on rent was seen intra-operatively. Meconiumstained liquor mixed with blood was seen in peritoneal cavity. The rent was extended laterally on both sides to deliver a live female child with a birthweight of $2.5 \mathrm{~kg}$ followed by the placental delivery by CCT and uterine repair with bilateral tubal ligation was done. Bladder was found to be intact. Estimated blood loss was $1200 \mathrm{ml}$. Postoperatively patient was transfused two units of blood. She reported COVID positive on post-operative day 1 and was managed accordingly as per the COVID protocols. Antibiotics were given for 7 days. Rest of the postoperative period remained uneventful. Patient was sent for home quarantine on post-operative day 5 with urinary catheter in situ.

\section{Case 2}

\section{Uterine rupture in primi with unscarred uterus}

A 21-year-old primigravida with breech presentation at a gestation of 34 weeks 6 days with preterm labour pains had been referred to our centre from a PHC. Patient was examined and a reactive CTG trace was obtained. She had adequate uterine contractions and a cervical dilatation of 3 $\mathrm{cm}$. Patient was explained the risks of vaginal breech delivery and consent for emergency LSCS was taken. Within 30 mins, she was taken up for LSCS under spinal anaesthesia. On entering the abdominal cavity, there was sudden gush of blood and placenta was found to be lying anterior to uterus, suggestive of rupture uterus and estimated haemo-peritoneum was around 1500-2000 ml.

After separating the placenta, a fresh stillborn male baby weighing $2.5 \mathrm{~kg}$ was delivered as breech extraction through the uterine defect. The defect was an inverted Tshaped rent in the upper segment extending up to the fundus, approximately $9-10 \mathrm{~cm}$. The lower uterine segment and bladder were intact. Uterine repair was done successfully. The estimated blood loss was 2000-2500 ml. Patient had received 6 units of blood and blood components post-operatively. Patient was explained prognosis for the subsequent pregnancies and was discharged uneventfully from the hospital on postoperative day 7 after stitch removal.

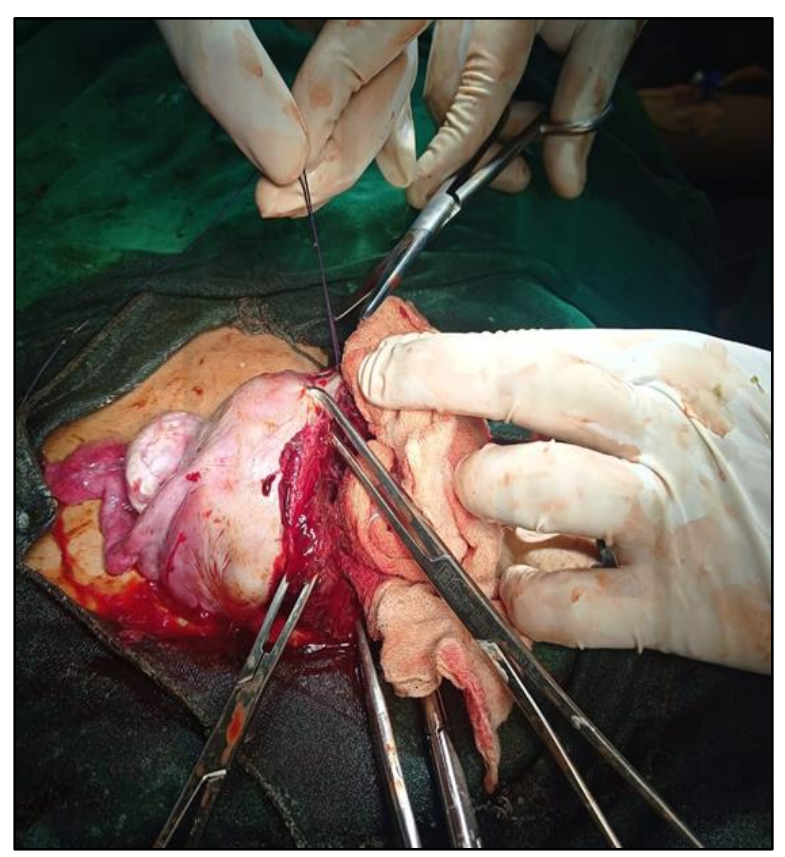

Figure 1: Rupture in an unscarred uterus in primigravida with breech presentation. 


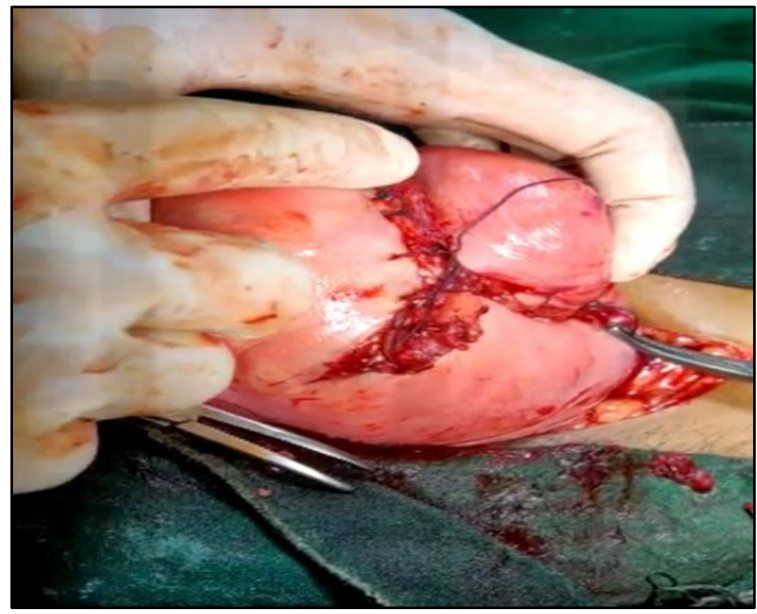

Figure 2: The uterine scar after repair.

\section{Case 3}

\section{Uterine rupture in grand multipara with fetal anomaly}

A 30-year-old grand multipara came to the emergency at a gestation of 38 weeks 3 days with ultrasound documented intrauterine fetal demise with fetal hydrocephalus and holoprosencephaly with leaking per vaginum for 24 hours and labour pains. She had three live female children, all born by institutional vaginal deliveries and history of stillbirth due to suspected congenital anomaly in the first pregnancy. On examination, fetal parts were felt superficially which was thought to be due to reduced amount of liquor and fetal heart could not be localised by the stethoscope. Patient had mild uterine contractions and a cervical dilatation of $6 \mathrm{~cm}$ but the station of head was at -3. Decision of decompression of hydrocephalus followed by trial of labour was taken. Patient was explained the risks and prognosis and a consent was obtained. An ultrasound was done which confirmed the above fetal anomalies and intra-uterine death. Transabdominal cephalocentesis for decompression of hydrocephalus was done under USG guidance and $300 \mathrm{~mL}$ of fluid was removed. On repeat per vaginal examination, cervical dilatation was still at $6 \mathrm{~cm}$ but the station had descended to -2 . Oxytocin was started at a low dose of $2 \mathrm{mU}$ per minute and escalated to $4 \mathrm{mU}$ per minute after an hour but no progress of labour was seen. After 4 hrs of Oxytocin drip at above mentioned rate patient had significant pallor and tachycardia with pulse rate of 120 beats per minute with cessation of uterine contractions while other parameters remained normal. Hence, decision for laparotomy under anaesthesia was taken in view of suspected rupture uterus and patient was shifted to OT. On opening the abdomen, baby was found lying in the peritoneal cavity. A stillborn male child with an enlarged head weighing $2.1 \mathrm{~kg}$ was delivered. An unsalvageable uterus was seen with a rupture in lower uterine segment and left lateral wall extending upto round ligament above and cervix below, with the placenta inside the cavity on the posterior wall. Bladder was seen adherent to the lower segment flap. Lower uterine segment was thin and friable but integrity was maintained and bladder dissection was done with difficulty. A peripartum subtotal hysterectomy with left salpingoophorectomy and right salpingectomy was done as rent was extending towards left broad ligament and ovary could not be salvaged. Estimated blood loss was $1300 \mathrm{ml}$. Patient received 2 units of blood post-operatively. Rest of the post-operative period was uneventful. Patient was discharged on post-operative day 8 after stitch removal.

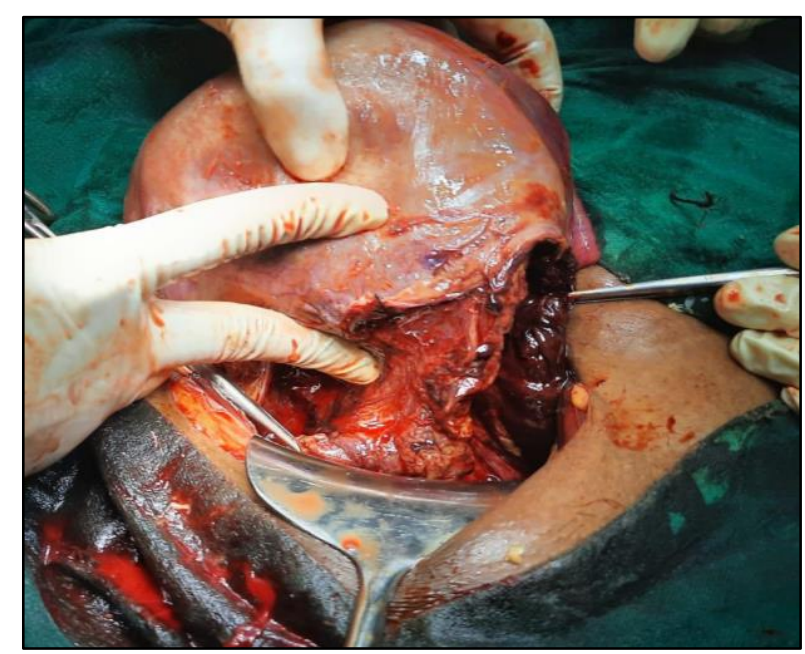

Figure 3: Rupture of lower uterine segment extending upto the left lateral wall seen in grand multipara with fetal hydrocephalus.

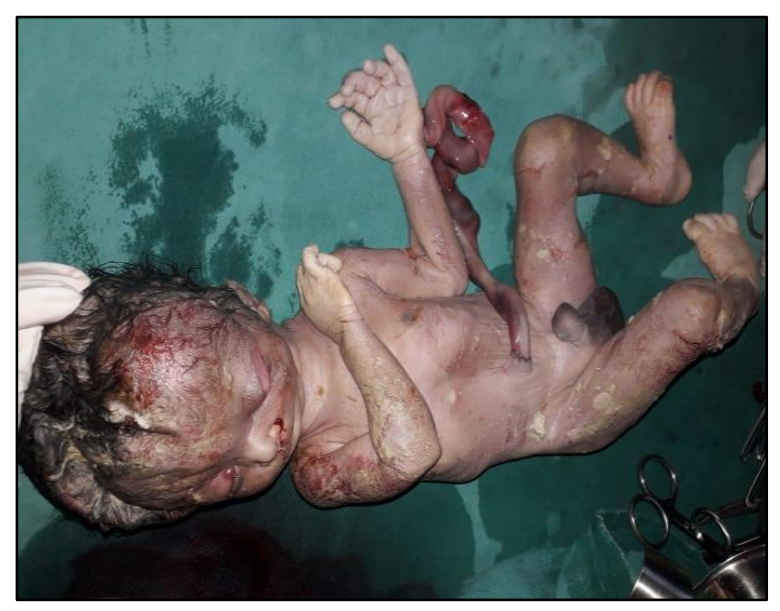

Figure 4: The dead fetus with enlarged head confirming the USG findings of hydrocephalus.

\section{DISCUSSION}

This rare complication of pregnancy is associated with life threatening complications to both mother and fetus. The above series suggest that the signs and symptoms of uterine rupture are usually variable and nonspecific, hence posing a challenge for the diagnosis. This often leads to a delay in diagnosis which causes significant fetal and maternal morbidity as well as mortality. More than threefourths of uterine rupture cases occur during term gestational age, as seen in two out of the three cases in the 
above series. ${ }^{5}$ The first case describes a woman with two prior caesarean delivery scars which has a $70 \%$ incidence of rupture as reported by the WHO multicountry survey on maternal and newborn health. ${ }^{6}$ But due to the atypical symptoms, the diagnosis could not be made before the abdomen was opened. Due to timely intervention and avidity of the obstetrician, both the fetus and mother had been saved.

The second case describes a case of rupture uterus in a primigravida with breech presentation at preterm gestation which is a rarity in itself. Although the malpresentation is a known risk factor for uterine rupture, it is very unusual unless there is a history of external cephalic version, induction of labour or trial of labour. In the rural setting, there is often a history of Dai handling which remains obscured but can be very pivotal to the diagnosis.

In the third case, grand multiparity with hydrocephalus and history of trial of vaginal delivery can be implicated as the probable causes of rupture. The hydrocephalus prevented the head descent and it also applied pressure on the lower segment with every contraction, ultimately leading to rupture. But due to the atypical presentation, diagnosis was slightly delayed but the patient was managed just in time to prevent any serious morbidity.

Difficult forceps delivery, breech extraction and unusual fetal enlargement such as hydrocephalus are also documented causes of uterine rupture as stated by Sudha et al in their study done in Tamil Nadu with $68.6 \%$ dead babies and $8.6 \%$ maternal mortality rate. ${ }^{7}$

Hence, rupture uterus can be very challenging due to the difficult diagnosis and variable presentation.

\section{CONCLUSION}

As seen in the day-to-day clinical scenario, uterine rupture can have varied presentations. Therefore, in order to be able to make an early diagnosis, the suspicion of uterine rupture must be kept in mind. Timely intervention like laparotomy can drastically reduce maternal and fetal morbidity as well as mortality. Patients must be encouraged for regular antenatal check-ups so that fetal malpresentations and anomalies can be identified in time and high-risk mothers can be made aware of the risk of rupture uterus. Most women with a previous uterine repair can have favourable outcome with tertiary level antenatal care and elective admission in subsequent pregnancies.

Funding: No funding sources

Conflict of interest: None declared

Ethical approval: Not required

\section{REFERENCES}

1. Togioka BM, Tonismae T. Uterine Rupture. In: StatPearls. Treasure Island (FL): StatPearls. Available at:

https://www.ncbi.nlm.nih.gov/books/NBK559209/. Accessed on 10 May 2021.

2. Herrera FA, Hassanein AH, Bansal V. Atraumatic spontaneous rupture of the non-gravid uterus. J Emerg Trauma Shock. 2011;4(3):439.

3. Al-Jufairi ZA, Sandhu AK, Al-Durazi KA. Risk factors of uterine rupture. Saudi Med J. 2001;22(8):702-4.

4. Gibbins KJ, Weber T, Holmgren CM, Porter TF, Varner MW, Manuck TA. Maternal and fetal morbidity associated with uterine rupture of the unscarred uterus. Am J Obstet Gynecol. 2015;213(3):382.e1-6.

5. Revicky V, Muralidhar A, Mukhopadhyay S, Mahmood T. A Case Series of Uterine Rupture: Lessons to be Learned for Future Clinical Practice. J Obstet Gynaecol India. 2012;62(6):665-73.

6. Motomura K. Incidence and outcome of uterine rupture among women with prior CS: WHO Multicountry survey on maternal and newborn health. Scientific Reports. 2020;7:44093.

7. Sudha N, Amulya MN. A study on maternal and fetal outcome in uterine rupture at a tertiary care hospital. Int J Clin Obstet Gynaecol. 2019;3(6):141-4.

Cite this article as: Aggarwal S, Jindal M, Minhas S. Case series on uterine rupture depicting the atypical presentations in the labour room. Int $\mathbf{J}$ Reprod Contracept Obstet Gynecol 2021;10:4549-52. 